\title{
Artritis reumatoide en Pediatría
}

\author{
Deș. González, Benito**; Batros, Tulío**: Rıbolíc-
} do, Liliana**; Sorenscn, Ricardo**.

La artritis remautoide (AR) es una enfermedad crónica, que comprometc grandes y pequeñas articulaciones, que presenta lesiones viscerales y del tejido conectivo, lo cual la da el carácter de enfermedad sistémica. $(1,2,3)$ La etiologia de la artritis remantoide es desconocida. En el niño se manifiesta con características elinicas distintas a las del adulto. (4) El diagnóstíco es difícil por el curso clínico variable y ausencia de hallazros de laboratorio patognomónicos.

En ol presente trabajo se expone la expcriencia de la Unidad de Inmunología del Hospital Luis Calvo Mackenna, considerando las dificultades de clasificación $y$ presentación clínica, exámenes de laboratorio y tratamiento de la enfermedad.

Se presenta, además, un estudió del llatuado Factor Antiperinuclear, descrito por Nienhuis y Mandema en 1964. (5, 6. 7) Este factor se encontró en el suero de pacientes adultos con AR. Reacciona con gránulos ritoplasmáticos de células epiteliales obtenidas ricl raspado bucal de individuos sanos. Estas colulas incubadas con suero de pacientes adultos y luego sometidas a un procedimiento de inmunofturescencia indirecta, presentan fluorescencia de tipo granular alrededor del núcleo, por lo cual

\footnotetext{
- Presentado como Relato Oficial en las V Tornadas Antuales de Ia Sociedad Chilena de Pediatria. Lo Barnechea, 1975.

* Unidad Inmunología H. Luis Calvo Mackenna.

* "Unidad Inmunología H. Luis Calvo Mackenna. Chile.
}

denominaron a este factor como Factor Antiperinuclear (APF).

La positividad del método, descrita por estos autores, alcanza a un $50 \%$, con una especificidad del $99 \%$. Este factor no se encuentra presente en otras patologías arliculares bi tampoco en otras mesenquimopatías. (5)

\section{MATERIAL Y METODO}

Los pacientes estudiados son 45 niños portadores de AR, de 3 a 15 años de edacl, controlados en la Linidad de Inmunología del Hospital Luis Calvo Mackeuna desde abrit dc 1972 a junio de 1975.28 corresponden a mujeres y 17 son varones.

Se clasificó a nuestros pacientes según lá forma de comienzo de la enfermedad, sigutiendo las siguientes normas:

1. Forma monoarticular: artritis de una articulación con una duración mayor de 3 meses.

2. Forma oligotrticular: comproniso de 2 a 5 articulaciones.

3. Forma poliarticular de comienzo brusco: compromiso poliarticular y, en plazo de días, compromiso del estado general. fjcbre, erupción cutânea.

4. Forma poliarticular de comicnzo insidioso: molestias generales arrastradas, tendencia a formación de nódulos e incapacidad fí. sica. $(8,2)$

Las recomendaciones propuestas por la Asociación Americana de Reumatología (ARA) 
para el diagnóstico defínitivo o probable, (9) son difíciles de aplicar en el estudio de la enfermedad en los niños, por los cambios radiológicos poco comunes, antecedentes anamnésticos difíciles de obtener, falta de estudio rutinario biópsico.

Los criterios empleados en nuestra serie para catalogar como definitiva o probable la enfermedad, fueton los expuestos por Grokoest y colaboradores. (10)

1. Poliartritis de un mínimo de 6 semanas, o menos de 6 semanas en presencia de cualquiera de los siguientes hallazgos: iritis, erupción cutánea, anquilosis, contracturas en flexión, debilidad muscular, anemia, leucocitosis de 20.000 o más, dolor en columna cervical con o sin cambios radiologicos.

2. Sintomas constitucionales: combinación de fiebre, debilidad y pérdida de peso.

3. Elevación de la velocidad de eritrosedimentación.

4. Exclusión de diagnósticos de fiebre reumática, LED, dermatomiositis, periarteritis nodosa, esclerodermia, artritis tbe, leucemia y linfoma.

Para el diagnóstico definitivo debe encontrarse todos los criterios anotados.

Se practico al ingreso: VHS, hemoglobina, recuento leucocitario, cuantificación de inmunoglobulinas, determinación de anticuerpos antinucleares (AAN), determinación de factor reumatoideo (Waaler Rose, más específico que la prueba del Latex), biopsia articular (en 3 pacientes), determinación del factor antiperinuclear en 20 pacientes.

En la selección del tratamiento hemos usado diversos esquemas, según la gravedad del caso. evitando en lo posible el tratamiento corticoidal.

Todos nuestros pacientes con attritis monoarticular y oligoarticular recibieron aspirina como único tratamiento. En las formas severas y de comienzo insidioso, se recurri 6 a corticoides y sales de oro.

En la anamnesis el antecedente de traumatismo se encontró en 3 casos y en 3 el de anigdalitis purulenta.

\section{RESULTADOS}

1. Edad y sexo. En general la AR es más frecuente en mujeres, excepto en los pacientes más pequeños, donde predominan los varones. Hay 2 zonas de mayor incidencia de la enfermedad, una comprendida entre los 2-4 años y la otra entre \&-11 años (Figura Nং 1).

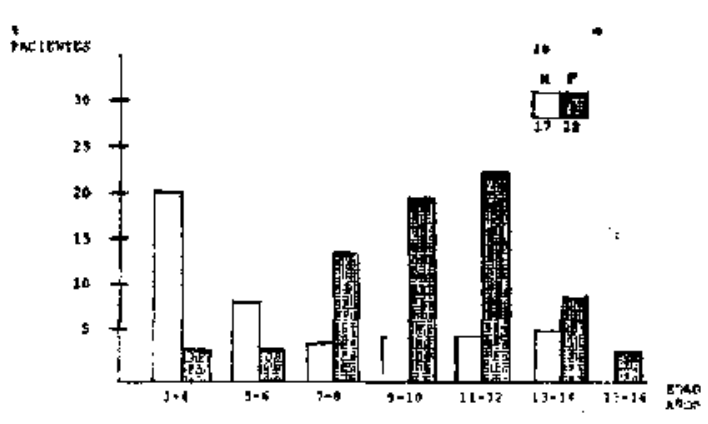

Figura N: 1

Distribución por edad y sexo en 45 casos de artritis retrmatoide.

2. Clasificación del material. E1 diagnóstico definitivo de la enfermedad se pudo establecer en el $34 \%$ de los casos de nuestra serie, quedando un $66 \%$ en la categorfa de AR probable. Los niños con mayor compromiso articular o sistémico tuvieron diagnósticos de certeza, no así las formas benignas, como las monoarticulares, que en un $100 \%$ quedaron con diagnostico probable y las oligoarticulares con $85 \%$ de diagnóstico probable (Tabla № 1).

3. Evolución clínica. Después de 2 años de evolución de la enfermedad, el $71 \%$ de los pacientes está asintomático. Un paciente fallece a los 4 años de edad. Inicib su enfermedad con poliartritis y gran compro. miso del estado general, y recibió prednisona y sales de oro. Al año de tratamiento hizo una hepatitis subaguda que fue la causa de su deceso.

La mortalidad total de nuestra serie es de 2,3\% (ver Tabla № 2).

La presencia de graves deformaciones articulares se observó en las formas poliarticulares de comienzo insidioso, especial- 
FORMA DE COMIENZO, DIAGNOSTICO DEFINITIVO Y PROBAALE EN 45

PACIENTES CON AR

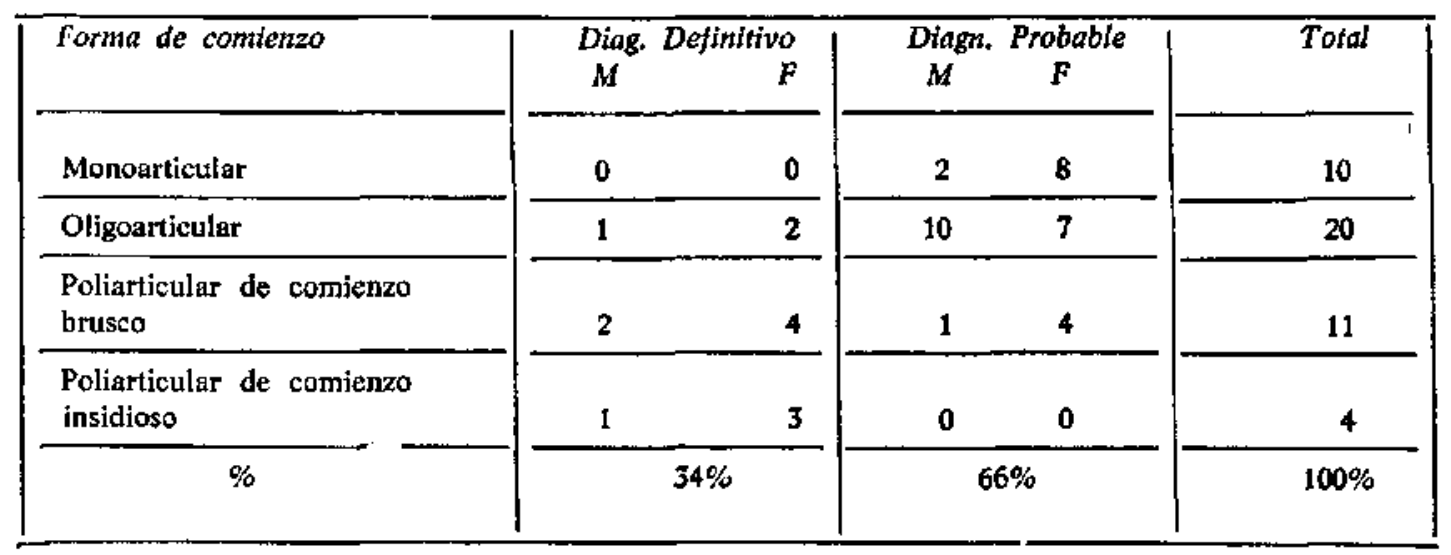

mente con compromiso de articulaciones interfalángicas. La evolución hacia estas deformaciones puede suceder en presencia de escasa sintomatología clínica.

4. Examenes de laboratorio. El grado de anemia, aumento de la velocidad de eritrosedimentación y leucocitosis, se correlaciona en buena forma con la gravedad del proceso. Sólo un $20 \%$ de nuestros pacientes tenía recuento de leucocitos superiores a $10.000 \times \mathrm{mm}^{3}$.

Destaca la negatividad del Factor Reumatoldeo (Waaler Rose) en un $96 \%$ de nuestros casos, y la presencia de anticuerpos antinucleares $(t)$ en un $60 \%$ en algún momento de la evolución. EI 34\% de los $\mathrm{AAN}(+)$ se encontró en títulos superiores a $1 / 100$, regresando a valores negativos en todos los casos en períodos de algunas semanas a meses de evolución.

Se pudo apreciar una disminución de IgA en un $8 \%$ de los pacientes, porcentaje significativamente meyor que el encontrado en la población general. Este déficit de IgA se asoció en enfermos con Waaler Rose(t).

EVOLUCION CLINICA EN 45 PACIENTES CON AR DESPUES DE 2 ANOS DE OBSERVACION

\begin{tabular}{|lccc|}
\hline Forma de comienzo & Asintomáticos & Sintomáticos & Fallecidos \\
\hline & 7 & 3 & \\
Monaarticular & 15 & 1 & \\
Oligoarticular & 9 & 3 & 1 \\
Poliarticular de comienzo brusco & 1 & 2 & \\
Poliarticular de comienzo insidloso & & 9 & 1 \\
\hline TOTAL & 32 & 9 \\
\hline
\end{tabular}


Las inmunoglobulinas IgG-IgM en un $12 \%$ se mantuvieron persistentemente elevadas y coincidiendo con la elevación el gtupo de mayor compromiso general.

Las formas benignas cursaron un $60 \%$ con Igs normales. Un $30 \%$ tuvo IgM clevada petsistentemente (Tabla № 3).

En relación a los anticuerpos antiperinucleates, ninguno de nuestros pacientes turo resultado $(t)$.

Pacientes adultos con AR delinitivo presentaron una positividad de $31 \%$, inferior a la descrita en la literatura. Comparado con el Waaler Rose, ambos tienen escasa positividad.

Los pacientes con compromiso mono u oligoarticular recibieron como tratamiento aspirina con evolución favorable en $66 \%$ de los casos.

El $28 \%$ de nuestros pacientes con compromiso poliarticular de comienzo insidioso $y$ brusco, recibió prednisona y en un $53 \%$ tuvo evolución satisfactoria, logrando períodos prolongados de remisión clínica.

El $16 \%$ recibió sales de oro, y no bicieron complicaciones hematológicas ni renales. Un caso se complicó con una dermatitis áurica que motivó la suspensión del tratamiento. No se observó mejores resultados que los obtenidos con terapia corticoidal.

En algunos casos se asoció ácido acetilsalicílico (60-100 mg x kg peso), aminopirina (15 $\mathrm{mg} \times \mathrm{kg}$ peso al día).

Tabla N? 3

NIVELES DE INMUNOGLOBULINAS SERICAS AL INGRESO EN 45 AR (mg\%)*

\begin{tabular}{|ll|c|c|c|c|}
\hline Formas de comienzo & $N *$ & $J g G$ & $I g M$ & $I g A$ \\
\hline Poliarticular de comienzo insidioso & & 4 & 2332 & 200 & 273 \\
\hline l'oliarticular de comienzo brukco & Def & 8 & 2155 & 204 & 259 \\
\hline Prob & 4 & 2170 & 267 & 217 \\
\hline Oligourticulils & Def & 3 & 1439 & 257 & 175 \\
\hline Monoarticular & Jrob & 16 & 1680 & 160 & 216 \\
\hline Normales & & 10 & 1704 & 234 & 186 \\
\hline
\end{tabular}

*Valores promedio en cadı grupo.

\section{CONCLUSIONES}

La artritis teumatoide entra en el campo de las enfermedades que tienen base inmunol6gica, desconociéndose el o los factores que motivan este comportamiento anómalo. En nuestros pacientes no se encontro $\mathrm{cl}$ antecedente de infección previa descrita por otros autores. (11)
La uttritis reumatoide es una entidad cuyo diagnóstico es esencialmente clínico. La positividad del Waaler Rose alcanza a un $4 \%$ en nuestra casuística muy distinta a la encontrada en el adulto. (12)

La búsqueda del factor antiperinuclear en nucstros pacientes no mostró la excelente positividad y especificidad descrita por autores holandeses en AR del adulto. Mostraría cierta 
utilidad (31\% positividad) en pacientes adultos con AR confirmada. Por lo cngomoso y complicado de la técnica, no lo recomendanos para la rutina hospitalaria.

La presencia de algunos elementos de autoinmunidad como el aumento de la AAN, obliga a descartar con certeza la presencia de un LED. $(13,14)$

Nuestra casuística sugicre, además, buscar un délicit de JgA asociado, en especial cuando se encuentra factor reumatoide positivo.

\section{SUMMARY}

The clinical features and laboratory findings of 45 children with rheumatoid arthritis are analized. 17 were males and 28 , females.

The age of onset has two peacks:

a) 3-4 years, with a high incidence of males affected, and b) 8-11 years, with a clear predominance of females.

Strickingly, a positive rheumatoid factor was found only in 4 children with an associatod IgA deficiency, which are described elsewhere in this issue.

A test for antiperinuclear antibodies was performed in all the children analized: none was found positive. The positivity of this test in adults with RA was of $310 \%$

\section{REFERENCIAS}

J. Anselt. B. M.: Still's disease. In Dixon, A. St. j. cd. Progress in clinical Rheumatology. Boston, Little, Brown \& Co., 1965, pp. 95-113.

2. Brewer, J. E.: Major Problems in Clinical Pediatries W. B. Saunders, 1970, pp. 1.j.

3. Ansell, 8. M., Bywaters, E. G. I.: Y'ed. Clin. North America, 10: 921, 1963.

4. Ansell, B. M., Bywaters, E. G. L.: Bull, Rheum. Dis., 9: 189-192, 1959.

5. Wienhuis, R. L. F.: Mardema, E.: An. Rheum. Dis. 23: 302-305, 1964.

6. Deicher, H. R. G., Holman, H. R, Kunkel, H. G.: Arthr: and Rheum. 3: 1-15, 1960.

7. Mandema, E., Pollak, V., Kark, R, M., Rezaian, J.: J. Lab. Clin. Med. 58: 337-352, 1961.

8. Boone, J. E., Balduin, J., Levine, C.: Ped. Clin. North America. 21: 885, 1974.

9. Ropes, M., Benneit, G. A., Cobb, S., Jucox, R. and Jessar, R. A.: J. Chron. Dis. 5: 630, 1957.

10. Groḱoest. A. W., Snyder, A. I., Schlaeger, K.: Juvenile Rheumatoid Artlritis, Boston, Little, Brow/l \& Co., 1962.

11. Bariand, P.: Am. J. Med. 54: 143-147.

12. Calabro, J. J., Marchesano, J. M.: New Engl. I. Med. 277: 696-699, 746-749, 1967.

13. Netty, R.E. Cassidy, J. T., Sullivan, D. B.: J. Pediat. 83: 386-389, 1973.

14. Esies, D. Christian, C. L.: Medicine. 50: 85-95, 1971. 